\title{
The potential for circular dichroism as an additional facile and sensitive method of monitoring low-molecular-weight heparins and heparinoids
}

\author{
Timothy R. Rudd"*; Mark A. Skidmore ${ }^{\text {*; }}$ Scott E.Guimond'; Joseph Holman'; Jeremy E. Turnbull'; Robert M. Lauder²; \\ David G. Fernig'; Edwin A. Yates' \\ ISchool of Biological Sciences, University of Liverpool, Liverpool, UK; ${ }^{2}$ School of Health and Medicine, Division of Biomedical and Life \\ Sciences, Lancaster University, Bailrigg, Lancaster, UK
}

\begin{abstract}
Summary
The ultraviolet circular dichroism (CD) spectra of commercial low-molecular-weight heparins, heparinoids and other anticoagulant preparations have been recorded between 180 and $260 \mathrm{~nm}$. Principal component analysis of the spectra allowed their differentiation into a number of groups related to the
\end{abstract}

\section{Keywords}

Circular dichroism, low-molecular-weight heparin, heparinoids, principal component analysis, quality control

\section{Introduction}

The linear anionic polysaccharide heparin, which is widely employed as an anticoagulant is composed of a major disaccharide repeat unit of 1,4 $O$-linked $\alpha$-L-iduronic acid and $\alpha$-D-glucosamine, in which the predominant substitution pattern is $2-O$-sulfation of the iduronate residues and $\mathrm{N}$ - and 6-O-sulfation of the glucosamine residues. A number of other substitutions are possible, including $\mathrm{N}$-acetylation and 3-O-sulfation in glucosamine and both non-sulfated iduronate and $\beta$-D-glucuronate can occur, providing considerable sequence heterogeneity (1). Recently, the issue of heparin quality control and monitoring has attracted attention following the observation of serious side-effects thought to have arisen from samples of full-length heparin tainted with over-sulfated chondroitin sulfate $(2,3)$. This has also been shown to be a problem affecting low-molecular-weight heparins (LMWHs). Indeed, alkaline catalysed $\beta$-elimination and $\mathrm{H}_{2} \mathrm{O}_{2}$ oxidative treatments, that are widely used to depolymerise heparin, also degraded the over-sulfated chondroitin sulfate contaminant (4) effectively concealing it in LMWH preparations.

Pharmaceutically, heparin acts by binding antithrombin III (AT), increasing its rate of factor Xa inactivitation by two to three orders of magnitude. The [Heparin:AT] complex inhibits several means of their production reflecting the structural changes introduced by each process. The findings suggest that $C D$ provides a complementary technique for the rapid analysis of heparin preparations.

Thromb Haemost 2009; 102: 874-878

procoagulant serine proteases in addition to factor $\mathrm{Xa}$, including factors $\mathrm{II}_{\mathrm{a}}$ (thrombin), IXa, XIa and XIIa. Among other factors, the catalytic activity of heparin is dependent on both chain length and the presence of "AGAIA" pentasaccharide sequences based on $\quad[--4) \quad \alpha$-D-GlcNS,6S (1-4) $\beta$-D-GlcA $\quad(1-4)$ $\alpha$-D-GlcNS,3S,6S (1-4) $\alpha$-L-IdoA2S (1-4) $\alpha$-D-GlcNS,6S (1--], a family of high-affinity binding sequences for AT that causes a conformational change resulting in increased inhibition of factor Xa. Approximately $30 \%$ of full-length heparin chains contain this high affinity sequence, but only $15-25 \%$ of LMWH chains. This sequence also forms the basis of the synthetic anticoagulant drug fondaparinux sodium (FS) (5). Effective catalysis of AT-mediated inhibition of factor IIa (thrombin) requires the presence of the high-affinity pentasaccharide sequence, but also that the saccharide be at least 18 (6) residues in length, because it must bind and bridge both AT and factor IIa. LWMHs have a molecular weight typically between $4-6.5 \mathrm{kDa}$ (their chain lengths vary from approximately 8 to 14 residues), while retaining considerable anti-Xa activity, but exhibiting attenuated anti-IIa activity, thereby providing the important ability to affect these two proteins either selectively or jointly. In recent years, unfractionated heparin has been replaced in many applications by LMHWs and heparinoids. 
LMWHs are produced by the depolymerisation of heparin chains. This can be achieved by a number of methods, including deaminative degradation using nitrous acid (7), scission by isoamyl nitrate (8), $\beta$-elimination under basic conditions, lysis by bacterially derived eliminase enzymes (9) and oxidative depolymerisation, for example, with hydrogen peroxide (10). These treatments can result in structural modifications in addition to depolymeristion, for example the introduction of anhydromannose terminal reducing end residues during nitrous acid-based cleavage and introduction of a characteristic 4,5 unsaturated non-reducing end uronic acid terminal residue following either enzymatic cleavage or $\beta$-elimination.

Circular dichorism $(\mathrm{CD}$; more properly termed electronic circular dichroism) is a powerful spectroscopic technique that is widely used to investigate protein secondary structure, but it has also been employed to probe the structure of heparin and other glycosaminoglycans (GAGs) $(11,12)$. The C-5 epimers of hexuronic acid (D-GlcA and L-IdoA) have significant CD spectra of opposite sign (13) and these residues are also major chiral chromophores in GAGs, including heparin. Heparin contains a relatively lower proportion (which is variable, being-around 20
$\%$ in porcine intestinal mucosal heparin) of $N$-acetyl groups than other GAGs. The CD spectra of heparin, related GAGs and chemically modified derivatives have a number of prominant features; a negative spectral feature at $\sim 210 \mathrm{~nm}$ arising from $\mathrm{n} \rightarrow \pi^{*}$ transitions in carboxylate groups (13), a positive feature at $\sim 190 \mathrm{~nm}$ containing transitions attributed to oxygen [glycosidic linkage, ring and hydroxyl] (14) and $N$-sulfate groups (15) as well as transitions [n $\rightarrow \pi^{*}$ and $\pi \rightarrow \pi^{*}$ ] in the $N$-acetyl (amide) group giving rise to spectral features at $\sim 210$ and $\sim 190 \mathrm{~nm}$ (16). However, the details of the CD spectra of GAGs have been found to be very sensitive to changes in the environment of the uronic acids in particular. These changes can be induced by altered substitution patterns or the binding of particular cations (11).

While the need to provide improved methods for the quality control and monitoring of heparin has been highlighted by recent concerns over contaminants, the practical challenge of easily differentiating between heparin originating from distinct origins, via particular methods of production, or comparing batch to batch variation for quality control purposes using one relatively simple technique, is formidable. This can be tackled using nuclear magnetic resonance (NMR) spectroscopy $(17,18)$, but its
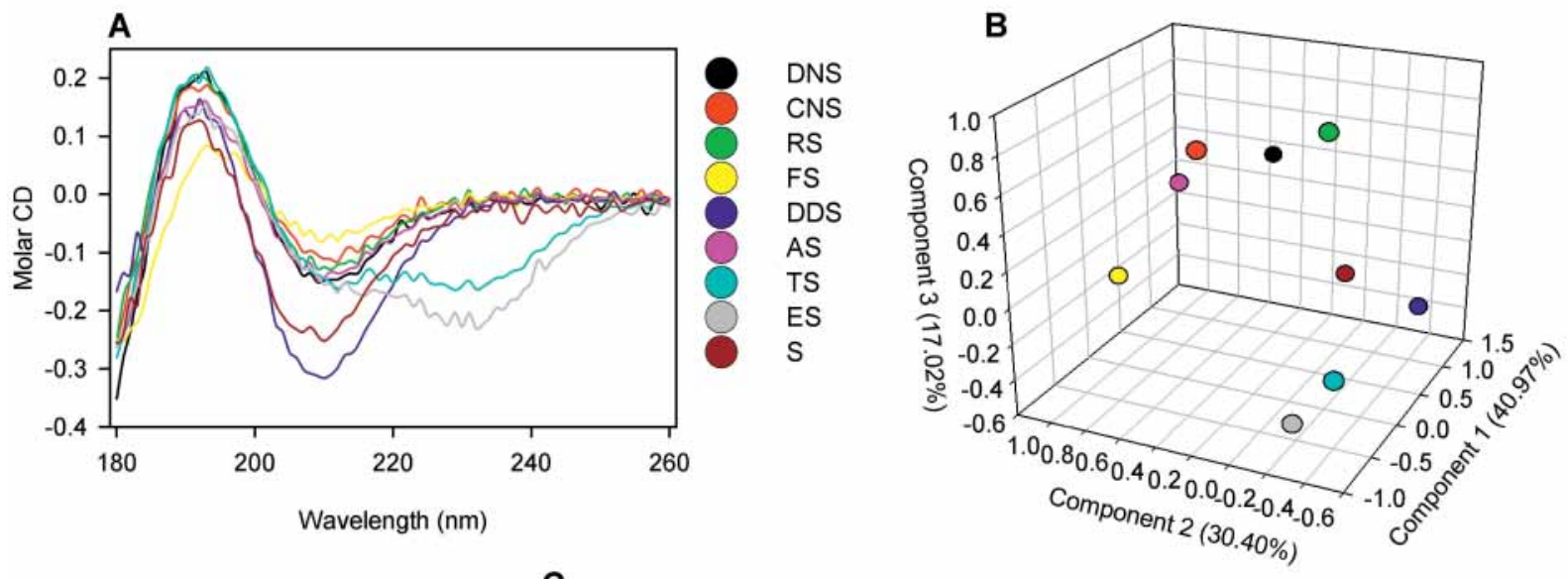

C
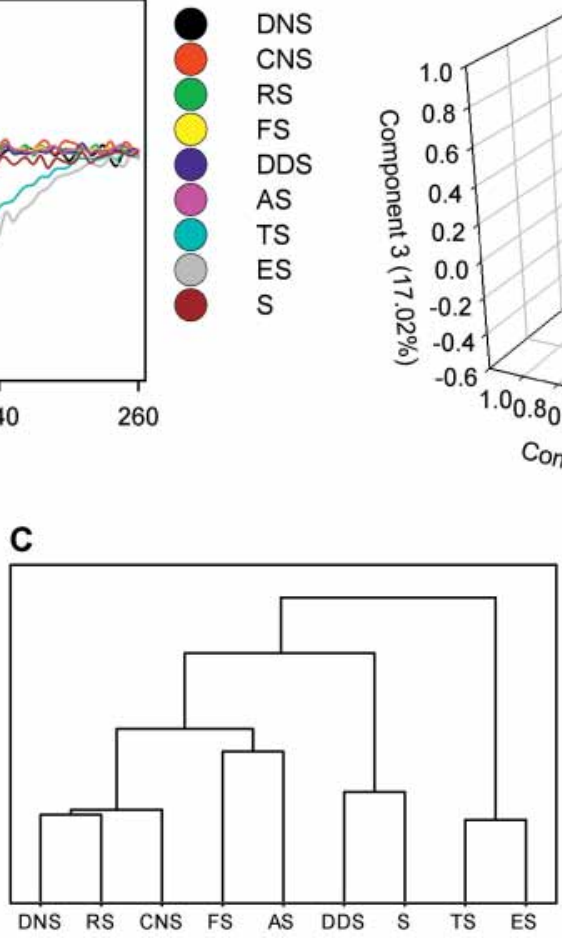

Figure I: CD spectra, loading plot and heirarchical cluster analysis of LMWHs and heparinoids. A) CD spectra of individual samples of LMWHs and heparinoids. B) Loading plot derived from PCA of mean-centred CD spectra of LMWHs and heparinoids. C) Heirarchical cluster analysis performed on the load plot derived from PCA of mean-centred CD spectra of LMWHs and heparinoids. The methods employed to prepare the samples were as follows: Nitrous acid degradation; dalteparin sodium (DNS) and reviparin sodium (RS). Synthesis; fon- daparinux sodium (FS). Cleavage with isoamyl nitrite; certaparin sodium (CNS). Oxidative depolymerisation with hydrogen peroxide; ardeparin sodium (AS) and sulfodexide (S); $\beta$-elimination with base; enoxaparin sodium (ES) and lyase enzyme degradation; tinzaparin sodium (TS). Note that danaparoid sodium (DPS) and sulodexide are both heparinoids containing, in the case of DPS, predominantly low-molecular-weight HS with DS and CS and in the case of sulodexide, predominantly LMWH with DS. 
relative complexity and expense still leaves room for additional and complementary methods. It has also been shown recently that $\mathrm{CD}$ is able to differentiate GAGs effectively (12) and does so on the basis of the distinct physical processes that are complementary to NMR.

The analysis of the CD spectra of GAGs has been achieved here through the application of multivariate analysis, a powerful statistical tool, which has been used to extract relationships from many forms of spectra and numerical data relating to GAGs (11, $12,19)$. The application of multivariate analysis permits spectra to be systematically compared and their similarities and differences to be evaluated (12,20,21).

We demonstrate that CD can be used to distinguish LMWHs in terms of the means employed in their production and also differentiate these from heparinoids containing other GAGs. Furthermore, the ability of $\mathrm{CD}$ to differentiate between these and other GAGs, including dermatan and chondroitin sulfate is shown.

\section{Methods and materials}

\section{SRCD}

SRCD spectra were recorded on beamline 12.1 at Daresbury Laboratory, a purpose-built synchrotron radiation circular dichroism (SRCD) facility, using a quartz sample cell (Hellma; 0.02 $\mathrm{cm}$ path length, $1 \mathrm{~nm}$ resolution), from 260 to $180 \mathrm{~nm}$. Spectra were recorded (at $10 \mathrm{mg} / \mathrm{ml}$ ) relative to $(+)$-10-camphorsulfonic acid $(1.0 \mathrm{mg} / \mathrm{ml})$.

\section{Factor analysis}

Factor analysis is used to uncover the latent structure (dimensionality) of a dataset and reduces a large number of variables to a smaller number of factors encapsulating the crucial informa- tion, while eliminating the noise. Consider a dataset $x_{i j}$ (cases $i$ and variables $j,(i=1, \ldots, n ; j=1, \ldots, k))$, factor analysis allows $x_{i j}$ to be expressed as a combination of $r$ factors, $\xi$. $x_{i j}=\lambda_{j 1} \xi_{i 1}+\lambda_{j 2} \xi_{i 2}+, \ldots,+\lambda_{j i} \xi_{i r}+\sigma_{i j}$. The $\lambda$ terms correspond to the factor loadings to be estimated and $\sigma_{\mathrm{ij}}$ is the measurement error in $\mathrm{x}_{\mathrm{ij}}$, or is the part of $\mathrm{x}_{\mathrm{ij}}$ that is not accounted for by the $\mathrm{r}$ underlying factors. In matrix notation, this representation can be expanded to; $\mathrm{X}=\Pi \Gamma^{\prime}+\Delta, \mathrm{X}$ (n by k matrix) - the observed cases, $\Pi$ (n by $\mathrm{r}$ matrix) - the scores on the underlying factors, $\Gamma$ ' ( $\mathrm{r}$ by k matrix) - the transpose of the factor loadings, and $\Delta$ ( $\mathrm{n}$ by k matrix) - the measurement errors. The most common form of FA is principal component analysis (PCA). PCA performs the optimum co-ordinate rotation to align the axes so that the variance within the data is maximised, therefore transforming a set of correlated variables into a set of uncorrelated variables. The most common form of rotation is the varimax rotation, which seeks to minimise the complexity of the components by maximising the larger loadings and minimising the smaller loadings within each factor/component. These uncorrelated variables are linear combinations of the original variables and the linear combination which extracts the maximum variance from the variables is the first principal component. Once this is found it is removed and another set of linear combinations, which explains the maximum proportion of the remaining variance is found, providing the second principal component. This continues until all the variance of the dataset can be explained. There can be as many components as variables (22).

\section{Multivariate analysis}

Multivariate analysis was performed using SPSS 15.0 (SPSS UK Ltd., Woking, UK), with prior mean-centering. Factor analysis was performed, with components extracted by PCA with a correlation matrix. The components were then rotated using the varimax method. Cluster analysis was performed using Minitab
Figure 2: Multivariate analysis of $C D$ spectra of LMWHs and other GAGs. A) CD spectra of LMWHs, heparinoids, CS and DS. B) Load plot derived from $P C A$ of mean-centred $C D$ spectra of LMWHs, heparinoids, CS and DS spectra. C) Cluster analysis performed on the load plot. Additional GAGs included in this analysis were all high-molecularweight samples; heparin from porcine intestinal mucosa, CS from bovine trachea, DS and chondroitin sulfate from porcine trachea.
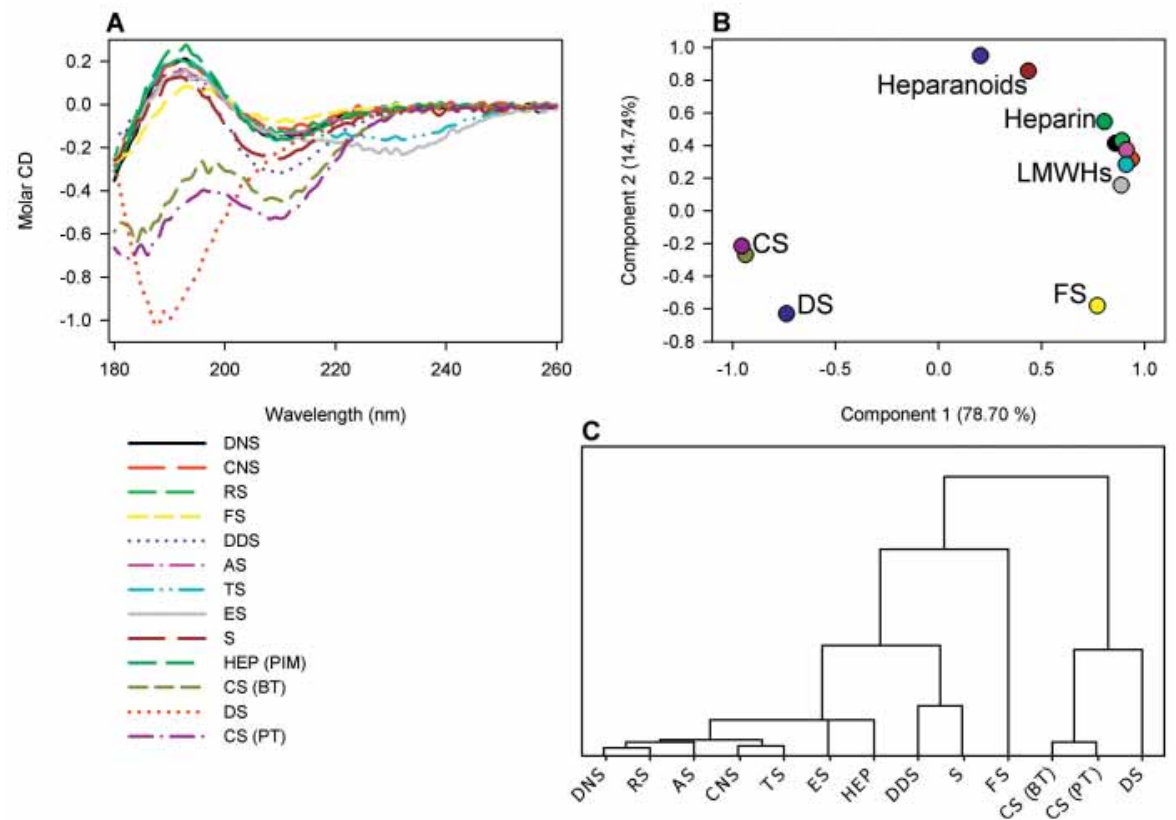
(Minitab Ltd., Coventry, UK) with single linkage. Euclidean distances were measured and clusters were determined by $80 \%$ similarity.

\section{Definitions}

Components represent the underlying dimensions that summarise or account for the original set of observed data. Component loadings are the correlation coefficients between the variables (rows) and factors (columns), the squared factor loadings indicate the percentage of the variance in the original variable that is explained by a factor. Component scores are a composite measure created for each observation on each factor extracted in the factor analysis.

\section{Results}

\section{Circular dichroism differentiates LMWH samples in terms of their method of production and can distinguish LMWHs and heparinoids}

The heparin CD spectra were of the expected form, with a positive peak at approximately $190 \mathrm{~nm}$ and a negative peak at 210 $\mathrm{nm}$. For eight of the spectra the positive peak arose at $192 \mathrm{~nm}$. These eight were split into two groups, the first consisted of dalteparin sodium (DNS), certaparin sodium (CS), reviparin sodium (RS) and tinzaparin sodium (TS), and the second contained danaparoid sodium (DPS), ardeparin sodium (AS), enoxaparin sodium (ES) and sulodexide. The final sample, FS, was distinct from the other eight samples in that its positive band arose at 195 $\mathrm{nm}$. The positive band at $190 \mathrm{~nm}$ can be attributed to electronic transitions in oxygen atoms (ring, glycosidic linkage and hydro$\mathrm{xyl}$ ). Regarding the negative band at $210 \mathrm{~nm}$, the spectra fell into three groups, the first contained FS, again being distinct, the second contained the non-CS/DS containing samples, while the third contained the heparanoids; DS and sulodexide containing CS/DS. The increased magnitude of the peak at $210 \mathrm{~nm}$ for the heparanoids can be explained by the presence of $\mathrm{CS}$, which has a strong negative feature at $210 \mathrm{~nm}$ (Fig. 2A), arising from the $\mathrm{N}$-acetyl groups, which became more prominant following the addition of uniformally $\mathrm{N}$-acetylated CS to the heparin sample, and which contained a low proportion of $\mathrm{N}$-acetyl groups. The most distinct feature was a negative band at $230 \mathrm{~nm}$, found only in ES and TS, which were depolymerised by $\beta$-elimination and lyase enzyme digestion, respectively, both of which result in the introduction of a 4,5 double bond $(\mathrm{C}=\mathrm{C})$ into the non-reducing end terminal uronate residue. With the exception of FS, which is unique in being the only sample to comprise a single chemical entity, the AGAIA pentasaccharide specific for ATIII; DS, CS, RS and AS are derived using methods which cut the heparin chain without introducing additional chromophores and comprise another distinct group.

The three components (Fig. 1B) used to differentiate the LMWHs and heparanoids by PCA explained $96.3 \%$ of the variance with the mean-centred data set; component 1 differentiated between the heparanoids and the LMWHs depolymerised by $\beta$-elimination or lyase enzyme digestion. The component score plot for component 1 showed that the spectral features which differentiated the two groups of samples were the strengthened minima at $210 \mathrm{~nm}$ for the heparanoids and the minima at $230 \mathrm{~nm}$, which were specific to the LMWHs depolymerised by $\beta$-elimination or lyase enzyme digestion. Component 2 differentiated FS from the rest of the LMWHs and heparanoids, the score plot for component 2 indicated that the shift in the maxima for FS from 192 to $195 \mathrm{~nm}$ and smaller minima at $210 \mathrm{~nm}$ were the differentiating features. Component 3 differentiated between samples depolymerised by nitrous acid and isoamyl nitrite and to a lesser extent, those depolymerised by oxidative cleavage, from heparanoids, LMWHs depolymerised by $\beta$-elimination or lyase enzyme digestion, and FS. The component score plot for component 3 indicated that the differentiating spectral features were the maxima at $192 \mathrm{~nm}$ (component plots and component score plots not shown).

\section{CD can also differentiate between LMWHs, heparinoids and other GAGs}

Spectra of two other naturally occurring glycosaminoglycans, CS and DS, were also analysed by PCA. The spectra and their analysis, shown in Figure 2, demonstrated that there was a clear contrast between the heparin-like spectra of the LMWHs and heparinoids and those of pure DS and CS. CD readily differentiated the pharmaceuticals from the full-length glycosaminoglycans, CS and DS. The structural modifications that occured due to depolymerisation of full-length heparin provided the means to monitor the identity and purity of LMWHs by circular dichroism spectroscopy and PCA.

\section{Conclusion and discussion}

CD spectroscopy between 180 and $260 \mathrm{~nm}$ is a useful additional tool to distinguish LMWH samples from heparinoids and both of these from other full-length GAGs. Furthermore, within the LMWH samples, CD can differentiate between samples that have been produced using particular depolymerisation techniques. The basis of the ability of $\mathrm{CD}$ to differentiate between these samples resides in its sensitivity to the introduction of chromophores such as additional N-acetyl groups, in the case of heparinoids, or the addition of these groups to even higher levels in the cases of CS and DS, or modified uronic acid residues containing double bonds, in the case of the products of enzymatic cleavage or $\beta$-elimination.

The ability of $\mathrm{CD}$ to achieve this lends itself to future applications in the quality control and monitoring of pharmaceutical grade heparin preparations. CD provides an additional and highly complementary method to other existing monitoring procedures, in that it detects particular physical parameters which are distinct to those detected by other techniques. CD is also highly sensitive to the presence of other chiral entities such as proteins and peptides, which are of obvious relevance to the quality control of heparin preparations.

The measurements made here were all recorded on a purpose-built synchrotron CD beamline (SRS, Daresbury, UK) but, with recent improvements in commercial CD instrumentation, could be obtained readily using standard laboratory CD instruments. The application of principal component analysis to the CD spectra permitted a systematic and objective comparison of the spectra to be made and lends itself to automation as well as the assembly of libraries of spectra. 


\section{Acknowledgements}

The authors thank the Wellcome Trust, MRC and BBSRC for funding. David Clarke and Alan Brown of Daresbury Laboratory are thanked for their assistance collecting CD spectra and the STFC for provision of facilities on the CD beamline 12.1 of the Synchrotron Radiation Source at Daresbury Laboratory, Warrington, UK.

\section{Abbreviations}

AT, antithrombin III; DDS, danaparoid sodium; DNS, dalteparin sodium; CNS, certaparin sodium; RS, reviparin sodium; FS, fondaparinux sodium; TS, tinzaparin sodium; ES, enoxaparin sodium; AS, ardeparin sodium; S, sulodexide; HS, heparan sulfate; CS, chondroitin sulfate; DS, dermatan sulfate; PCA, principal component analysis; HEP, heparin; BT, bovine tracheal; PT, porcine tracheal; PIM, porcine intestinal mucosal.

\section{References}

1. Esko JD, Lindahl U. Molecular diversity of heparan sulfate. J Clin Invest 2001; 108: 169-173.

2. Guerrini M, Beccati D, Shriver Z, et al. Oversulfated chondroitin sulfate is a contaminant in heparin associated with adverse clinical events. Nature Biotechnol 2008; 26: 669-675.

3. Kishimoto TK, Viswanathan K, Ganguly T, et al. Contaminated heparin associated with adverse clinical events and activation of the contact system. New Engl J Med 2008; 358: 2457-2467.

4. Zhang Z, Weiwer M, Li B, et al. Oversulfated chondroitin sulfate: impact of a heparin impurity, associated with adverse clinical events, on low-molecular-weight heparin preparation. J Med Chem 2008; 51: 5498-5501.

5. Keam SJ, Goa KL. Fondaparinux sodium. Drugs 2002; 62: 1673-1685; discussion 1686-1677.

6. Petitou M, Herault JP, Bernat A, et al. Synthesis of thrombin-inhibiting heparin mimetics without side effects. Nature 1999; 398: 417-422.

7. Shively JE, Conrad HE. Stoichiometry of the nitrous acid deaminative cleavage of model amino sugar glycosides and glycosaminoglycuronans. Biochemistry 1970; 9: 33-43

8. Jeske W, Wolf H, Ahsan A, et al. Pharmacologic profile of certoparin. Expert opinion on investigational drugs 1999; 8: 315-327.
9. Linhardt RJ, Grant A, Cooney CL, Langer R. Differential anticoagulant activity of heparin fragments prepared using microbial heparinase. J Biol Chem 1982; 257: 7310-7313.

10. Liu Z, Perlin AS. Evidence of a selective free radical degradation of heparin, mediated by cupric ion Carbohydrate Res 1994; 255: 183-191.

11. Rudd TR, Guimond SE, Skidmore MA, et al. Influence of substitution pattern and cation binding on conformation and activity in heparin derivatives. Glycobiology 2007; 17: 983-993.

12. Rudd TR, Skidmore MA, Guimond SE, et al. Glycosaminoglycan origin and structure revealed by multivariate analysis of NMR and CD spectra. Glycobiology 2008; 19: 52-67.

13. Morris ER, Rees DA, Sanderson GR, Thom D. Conformation and Circular-Dichroism of Uronic Acid Residues in Glycosides and Polysaccharides. J Chem Soc -Perkin Transactions 1975; 2: 1418-1425.

14. Stevens ES, Morris ER, Rees DA, et al. Synchrotron light-source applied to measuring the vacuum ultraviolet circular-dichroism of heparin. J Am Chem Soc 1985; 107: 2982-2983.

15. Braud C, Vert M. Acid-base and chiroptical properties of N-desulfated heparin. Macromolecules 1985; 18: $856-862$.
16. Greenfield NJ, Fasman GD. Circular Dichroism of 3-Methylpyrrolidin-2-One. J Am Chem Soc 1970; 92 : 177-181.

17. Casu B, Guerrini M, Naggi A, et al. Characterization of sulfation patterns of beef and pig mucosal heparins by nuclear magnetic resonance spectroscopy. Arzneimittel-Forschung 1996; 46: 472-477.

18. Guerrini M, Guglieri S, Naggi A, et al. Low molecular weight heparins: structural differentiation by bidimensional nuclear magnetic resonance spectroscopy. Semin Thromb Hemost 2007; 33: 478-487.

19. Rudd TR, Skidmore MA, Guimond SE, et al. Sitespecific interactions of copper(II) ions with heparin revealed with complementary (SRCD, NMR, FTIR and EPR) spectroscopic techniques. Carbohydrate Res 2007; 343: 2184-2193.

20. Duarte IF, Barros A, Almeida C, Spraul M, Gil AM Multivariate analysis of NMR and FTIR data as a potential tool for the quality control of beer. J Agric Food Chem 2004; 52: 1031-1038.

21. Almeida C, Duarte IF, Barros A, et al. Composition of beer by ${ }^{1} \mathrm{H}$ NMR spectroscopy: Effects of brewing site and date of production. J Agric Food Chem 2006; 54: 700-706.

22. Jolliffe IT. Principal component analysis. $2^{\text {nd }}$ ed. ed. New York: Springer; 2002 